\title{
Fully Connected PLL Networks: How Filter Determines the Number of Nodes
}

\author{
Átila Madureira Bueno, André Alves Ferreira, \\ and José Roberto C. Piqueira \\ Departamento de Engenharia de Telecomunições e Controle, Escola Politécnica da Universidade de \\ São Paulo, Avenida Prof. Luciano Gualberto, travessa 3, n. 158, 05508-900 São Paulo, SP, Brazil \\ Correspondence should be addressed to José Roberto C. Piqueira, piqueira@lac.usp.br
}

Received 5 October 2008; Revised 30 January 2009; Accepted 2 March 2009

Recommended by Jerzy Warminski

Synchronization plays an important role in telecommunication systems, integrated circuits, and automation systems. Formerly, the masterslave synchronization strategy was used in the great majority of cases due to its reliability and simplicity. Recently, with the wireless networks development, and with the increase of the operation frequency of integrated circuits, the decentralized clock distribution strategies are gaining importance. Consequently, fully connected clock distribution systems with nodes composed of phase-locked loops (PLLs) appear as a convenient engineering solution. In this work, the stability of the synchronous state of these networks is studied in two relevant situations: when the node filters are first-order lag-lead lowpass or when the node filters are second-order low-pass. For first- order filters, the synchronous state of the network shows to be stable for any number of nodes. For second-order filter, there is a superior limit for the number of nodes, depending on the PLL parameters.

Copyright (C) 2009 Átila Madureira Bueno et al. This is an open access article distributed under the Creative Commons Attribution License, which permits unrestricted use, distribution, and reproduction in any medium, provided the original work is properly cited.

\section{Introduction}

Synchronization networks assure the correct temporal order of information processing in communication systems, computation and control and many commercial systems adopt a masterslave synchronization strategy due to its reliable behavior, construction facility, and low cost [1-3]. In this architecture, a master node dictates the phase and frequency scales for the entire network.

On the other hand, in a fully connected architecture, phase and frequency scales are determined by all the nodes as they are all connected to each other. The main advantage of the fully connected architecture is its robustness when nodes are added or dropped [4]. Nowadays, besides being used in telecommunication networks, the fully connected 
architecture is used in digital electronic circuits for clock signal distribution [5-7], and in synchronous neural networks for pattern recognition [8-10].

Modeling fully connected networks result in nonlinear coupled oscillations. Some interesting works about this kind of architecture were developed between 1960 and 1980 [1115]. These papers were written when the service integration started to be taken into account for telecommunication networks and present several robust results for clock distribution systems, mainly considering linear analogous phase detection and linear coupling between nodes.

In these works, synchronous state frequencies and their stability conditions for fully connected PLL networks were presented depending on the free-running frequencies and node phase detector gains.

Trying to extend the results to the case with nonlinear coupling, in [16, 17], expressions for the synchronous state and conditions for its stability are derived, considering analog phase-detectors and first-order lag low-pass filters. The synchronous state of a fully connected network showed to be locally asymptotically stable for any number $N$ of nodes.

As, sometimes, double-frequency jitter degrades the performance of the PLLs [18-20], filters are designed more accurately [21, 22].

Here, a nonlinear state space model of fully connected PLL networks with $N$ nodes, considering a first-order lead-lag low-pass and a second-order low-pass filters in the nodes is developed, trying to minimize double-frequency distortion [23, 24].

First, models for the fully connected PLL network with first-order lag-lead node filters and second-order low-pass node filters are developed. Then, by using Taylor series development around the synchronous state [25], it was shown that for the lag-lead node filters, the synchronous state is locally asymptotically stable for any number of nodes, in the same way of the lag node filters. For third-order node filters, a local Hopf bifurcation that appears in the single node dynamics [26-29] gives a superior limit for the number of nodes in the network.

The contribution is about analytic modeling of this kind of network and experimental and numerical works can be conducted trying to confirm the results as suggested in $[27,30-$ 32].

\section{Fully Connected PLL Network Model}

In a fully connected PLL network with $N$ nodes, each node has $N-1$ inputs corresponding to the phase comparisons, as shown in Figure 1, with the nodes mutually coupled by using two-way connections $[30,31]$. As there is no integrated circuit with this architecture, the implementation of the nodes requires a combination of $N-1$ PLL chips with their phase detector outputs weighted and being the input of the filter $f^{(j)}(t)$ that feeds the local VCO $[17,30-32]$.

For a given node $j$, the output $v_{o}^{(j)}(t), j=1,2, \ldots, N$, and input signals $v_{o}^{(\ell)}(t), \ell=$ $1, \ldots, j-1, j+1, \ldots, N$, are given by

$$
\begin{gathered}
v_{o}^{(\ell)}\left(t-\tau_{\ell, j}\right)=\mathrm{v}_{o}^{(\ell)} \sin \left(\omega_{M}\left(t-\tau_{\ell, j}\right)+\theta_{o}^{(\ell)}\left(t-\tau_{\ell, j}\right)\right), \\
v_{o}^{(j)}(t)=\mathrm{v}_{o}^{(j)} \cos \left(\omega_{M} t+\theta_{o}^{(j)}(t)\right)
\end{gathered}
$$


where $\mathrm{v}_{o}^{(\ell)}$ and $\mathrm{v}_{o}^{(j)}$ are the amplitudes of the input and output signals, respectively; $\omega_{M}$ is the free-running angular frequency of all VCOs, $\theta_{o}^{(j)}(t)$ is the instantaneous phase of node $j$, $\theta_{o}^{(\ell)}\left(t-\tau_{\ell, j}\right)$ is the phase of node $\ell$ delayed by the signal propagation time from node $\ell$ to $j$, $\tau_{\ell, j}$

Assuming that all VCOs in the network have the same free-running angular frequency, the phase and frequency spatial errors can be defined as

$$
\begin{aligned}
& \vartheta^{(\ell, j)}(t)=\theta_{o}^{(\ell)}\left(t-\tau_{\ell, j}\right)-\theta_{o}^{(j)}(t), \\
& \dot{\vartheta}^{(\ell, j)}(t)=\dot{\theta}_{o}^{(\ell)}\left(t-\tau_{\ell, j}\right)-\dot{\theta}_{o}^{(j)}(t) .
\end{aligned}
$$

Considering the expressions of the phase errors between node $j$ and of all other nodes as given by (2.3) and summing them, the estimation of phase $\theta_{o}^{(j)}(t)$, of the node $j$, is written as follows:

$$
\theta_{o}^{(j)}(t)=\frac{1}{N-1} \sum_{\substack{\ell=1 \\ \ell \neq j}}^{N}\left(\theta_{o}^{(\ell)}\left(t-\tau_{\ell, j}\right)-\vartheta^{(\ell, j)}(t)\right) .
$$

The PLLs are described by a differential equation of order $P^{(j)}+1$, considering that the order of the linear filter $f(t)$ is $P^{(j)}[21,22]$. The transfer function $F^{(j)}(s)$ of the node $j$ filter can be expressed in the form of a rational fraction of polynomials:

$$
F^{(j)}(s)=\frac{N^{(j)}(s)}{D^{(j)}(s)},
$$

with

$$
\begin{aligned}
& N^{(j)}(s)=\sum_{m=0}^{M^{(j)}} \alpha_{m}^{(j)} s^{m}, \\
& D^{(j)}(s)=\sum_{p=0}^{P^{(j)}} \beta_{p}^{(j)} s^{p},
\end{aligned}
$$

considering that $M^{(j)} \leq P^{(j)}$.

A consequence from the fact that the filter can be modeled by (2.6) and from the structure of (2.1) and (2.2) is that the network dynamics can be expressed in a simpler way 


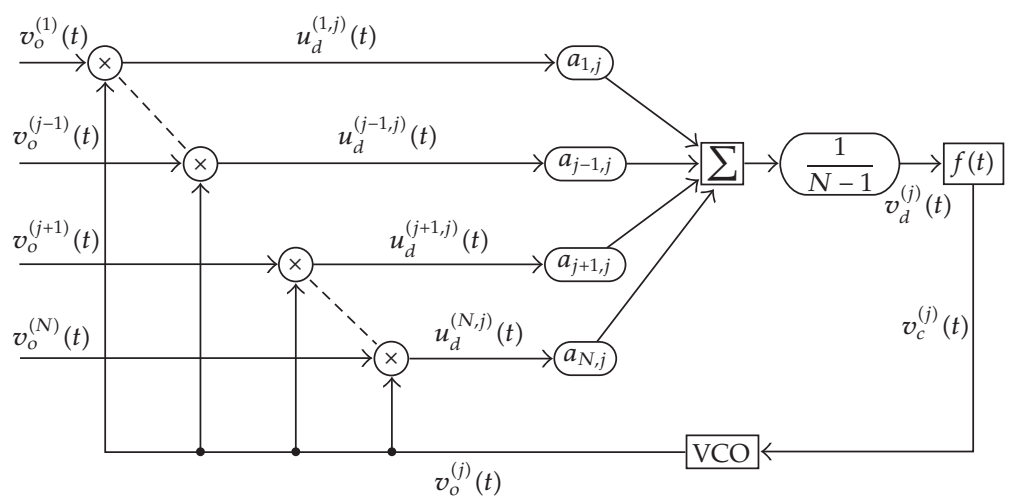

Figure 1: PLL block diagram in a fully connected architecture.

considering the operators $Q^{(j)}[\cdot]$ and $L^{(j)}[\cdot]$, and the node gains $G^{(\ell, j)}$ as follows:

$$
\begin{gathered}
Q^{(j)}[\cdot]=\sum_{m=0}^{M^{(j)}} \alpha_{m}^{(j)} \frac{d^{m}}{d t^{m}}(\cdot), \\
L^{(j)}[\cdot]=\sum_{p=0}^{P^{(j)}} \beta_{p}^{(j)} \frac{d^{p+1}}{d t^{p+1}}(\cdot), \\
G^{(\ell, j)}=\frac{1}{2} a_{\ell, j} k_{m}^{(j)} k_{o}^{(j)} \mathrm{v}_{o}^{(j)} \mathrm{v}_{o}^{(\ell)} .
\end{gathered}
$$

In these definitions, the positive numbers $k_{m}^{(j)}$ are the phase detector gains, $k_{o}^{(j)}$, the VCO control gains and $a_{\ell, j}$, the weighting factors between nodes $\ell$ and $j$.

Therefore, the filter inputs are

$$
v_{d}^{(j)}(t)=\frac{1}{N-1} \sum_{\substack{\ell=1 \\ \ell \neq j}}^{N} a_{\ell, j} u_{d}^{(\ell, j)}(t)
$$

with

$$
\frac{1}{N-1} \sum_{\substack{\ell=1 \\ \ell \neq j}}^{N} a_{\ell, j}=1
$$

The outputs of the phase detectors are proportional to the product between their input and output signals:

$$
u_{d}^{(\ell, j)}(t)=k_{m}^{(j)} v_{o}^{(\ell)}\left(t-\tau_{\ell, j}\right) v_{o}^{(j)}(t)
$$


resulting

$$
u_{d}^{(\ell, j)}(t)=\frac{G^{(\ell, j)}}{a_{\ell, j} k_{o}^{(j)}} u_{\delta}^{(\ell, j)}(t)
$$

with

$$
u_{\delta}^{(\ell, j)}(t)=\sin \left(\vartheta^{(\ell, j)}(t)-\omega_{M} \tau_{\ell, j}\right)+\sin \left(2 \omega_{M} t+\theta_{o}^{(\ell)}\left(t-\tau_{\ell, j}\right)+\theta_{o}^{(j)}(t)-\omega_{M} \tau_{\ell, j}\right) .
$$

Solving (2.3) for $\theta_{o}^{(j)}(t)$, replacing the result in (2.14), and doing some algebra:

$$
u_{\delta}^{(\ell, j)}(t)=\sin \left(\vartheta^{(\ell, j)}(t)-\omega_{M} \tau_{\ell, j}\right)+\sin \left(2\left(\omega_{M} t+\theta_{o}^{(\ell)}\left(t-\tau_{\ell, j}\right)\right)-\vartheta^{(\ell, j)}(t)-\omega_{M} \tau_{\ell, j}\right) .
$$

The output phase $\theta_{o}^{(j)}(t)$ of the VCO is controlled according to

$$
\frac{d}{d t} \theta_{o}^{(j)}(t)=k_{o}^{(j)} v_{c}^{(j)}(t)
$$

with control signal $v_{c}^{(j)}$ being the filter response to the weighted error signal $v_{d}^{(j)}$, and given by

$$
v_{c}^{(j)}(t)=f^{(j)}(t) * v_{d}^{(j)}(t)
$$

Consequently, replacing (2.17) in (2.16) and applying the convolution theorem [33]:

$$
k_{o}^{(j)} N^{(j)}(s) V_{d}^{(j)}(s)=s D^{(j)}(s) \Theta_{o}^{(j)}(s) .
$$

Considering the operators $Q^{(j)}[\cdot]$ and $L^{(j)}[\cdot]$ and using (2.5), (2.10), and (2.13),

$$
L^{(j)} \sum_{\substack{\ell=1 \\ \ell \neq j}}^{N} \vartheta^{(\ell, j)}(t)+Q^{(j)} \sum_{\substack{\ell=1 \\ \ell \neq j}}^{N} G^{(\ell, j)} u_{\delta}^{(\ell, j)}(t)=L^{(j)} \sum_{\substack{\ell=1 \\ \ell \neq j}}^{N} \theta_{o}^{(\ell)}\left(t-\tau_{\ell, j}\right) .
$$

Replacing (2.15) in (2.19)

$$
\begin{aligned}
& L^{(j)} \sum_{\substack{\ell=1 \\
\ell \neq j}}^{N} \vartheta^{(\ell, j)}(t)+Q^{(j)} \sum_{\substack{\ell=1 \\
\ell \neq j}}^{N} G^{(\ell, j)} \sin \left(\vartheta^{(\ell, j)}(t)-\omega_{M} \tau_{\ell, j}\right) \\
& \quad=-Q^{(j)} \sum_{\substack{\ell=1 \\
\ell \neq j}}^{N} G^{(\ell, j)} \sin \left(2\left(\omega_{M} t+\theta_{o}^{(\ell)}\left(t-\tau_{\ell, j}\right)\right)-\vartheta_{o}^{(\ell, j)}(t)-\omega_{M} \tau_{\ell, j}\right)+L^{(j)} \sum_{\substack{\ell=1 \\
\ell \neq j}}^{N} \theta_{o}^{(\ell)}\left(t-\tau_{\ell, j}\right),
\end{aligned}
$$

for $j=1,2, \ldots, N$. 
Finally, (2.20) models the fully connected PLL network, neglecting the secondharmonic term that is supposed to be filtered $[3,23,24]$. To simplify the analytical reasoning, for a given node $j$, it is considered that all the phase errors are equally weighted, and that all the constitutive parameters of the nodes are the same. These assumptions do not change the main conclusions as weight criterion and node parameters affect only the system transient behavior. Therefore, from now on $G^{(\ell, j)}=G, L^{(j)}[\cdot]=L[\cdot], Q^{(j)}[\cdot]=Q[\cdot]$ and

$$
L \sum_{\substack{\ell=1 \\ \ell \neq j}}^{N} \vartheta^{(\ell, j)}(t)+G Q \sum_{\substack{\ell=1 \\ \ell \neq j}}^{N} \sin \left(\vartheta^{(\ell, j)}(t)-\omega_{M} \tau_{\ell, j}\right)=L \sum_{\substack{\ell=1 \\ \ell \neq j}}^{N} \theta_{o}^{(\ell)}\left(t-\tau_{\ell, j}\right) .
$$

The fixed transmission delays, $\tau_{\ell, j}$, are also neglected, since they only shift the equilibrium points from its original position in equation not affecting their stability conditions (2.21). Using (2.5)

$$
-(N-1) L \theta_{o}^{(j)}(t)+G Q \sum_{\substack{\ell=1 \\ \ell \neq j}}^{N} \sin \left(\vartheta^{(\ell, j)}(t)\right)=0,
$$

for $j=1,2, \ldots, N$.

From (2.5) it can be seen that, in the synchronous state the phase of node $j$ is linearly dependent on the phase output of all the other nodes. Then, it is necessary to obtain a set of $N-1$ linearly independent state variables [31].

This is obtained by rewriting (2.22) for node 1 and for the other nodes as follows:

$$
-(N-1) L \theta_{o}^{(1)}(t)+G Q \sum_{\ell=2}^{N} \sin \left(\vartheta^{(\ell, 1)}(t)\right)=0,
$$

for node 1 , and

$$
-(N-1) L \theta_{o}^{(j)}(t)+G Q \sum_{\substack{\ell=1 \\ \ell \neq j}}^{N} \sin \left(\vartheta^{(\ell, j)}(t)\right)=0,
$$

for $j=2, \ldots, N$.

Expressing the phase difference between all the other nodes and the node 1, that is, subtracting (2.24) from (2.23), and considering (2.3) with $\tau_{\ell, j}=0$

$$
(N-1) L \vartheta^{(j, 1)}(t)+G Q \sum_{\ell=2}^{N} \sin \left(\vartheta^{(\ell, 1)}(t)\right)-\sum_{\substack{\ell=1 \\ \ell \neq j}}^{N} \sin \left(\vartheta^{(\ell, j)}(t)\right)=0 .
$$

Considering that

$$
\vartheta^{(1, j)}(t)=\theta_{o}^{(1)}(t)-\theta_{o}^{(j)}(t)=-\left(\theta_{o}^{(j)}(t)-\theta_{o}^{(1)}(t)\right)=-\vartheta^{(j, 1)}(t)
$$


and that

$$
\vartheta^{(\ell, j)}(t)=\theta_{o}^{(\ell)}(t)-\theta_{o}^{(j)}(t)=\theta_{o}^{(\ell)}(t)-\theta_{o}^{(1)}(t)+\theta_{o}^{(1)}(t)-\theta_{o}^{(j)}(t)=\vartheta^{(\ell, 1)}(t)-\vartheta^{(j, 1)}(t),
$$

working on (2.25),

$$
L \vartheta^{(j, 1)}(t)+2 \mu_{1} Q \sin \left(\vartheta^{(j, 1)}(t)\right)+\mu_{1} Q \sum_{\substack{\ell=2 \\ \ell \neq j}}^{N}\left(\sin \left(\vartheta^{(\ell, 1)}(t)\right)-\sin \left(\vartheta^{(\ell, 1)}(t)-\vartheta^{(j, 1)}(t)\right)\right)=0,
$$

for $j=2, \ldots, N$, and

$$
\mu_{1}=\frac{G}{N-1}
$$

The set of $N-1$ equations given by (2.28) is the general model that describes the dynamical behavior of the phase differences for the network nodes. The use of the lag filter, as in $[16,17]$, simplifies the mathematical reasoning. However, this kind of filter is useful only when the bandwidth, that is, the difference between input signal frequencies and central operation frequency of the PLL does not need to be narrow [34].

In practical cases, when narrow bandwidth and large gain are simultaneously required, lead-lag filters with transfer functions given by

$$
F(s)=\frac{s+\alpha_{0}}{s+\beta_{0}}
$$

are used [34].

The second-order filter, with transfer function given by

$$
F(s)=\frac{s+\alpha_{0}}{s^{2}+\beta_{1} s+\beta_{0}}
$$

prevents spurious side-bands by reducing the influence of double-frequency jitter and transmission noise [21-24,34], in spite of provoking bifurcation [26, 27].

Considering a first-order lead-lag filter in each node of the PLL network and (2.28),

$$
\begin{aligned}
\ddot{\vartheta}^{(j, 1)}(t)+\beta_{0} \dot{\vartheta}^{(j, 1)}(t)+2 \mu_{1} \dot{\vartheta}^{(j, 1)}(t) \cos \left(\vartheta^{(j, 1)}(t)\right)+2 \alpha_{0} \mu_{1} \sin \left(\vartheta^{(j, 1)}(t)\right) \\
+\mu_{1} \sum_{\substack{\ell=2 \\
\ell \neq j}}^{N}\left(\dot{\vartheta}^{(\ell, 1)}(t) \cos \left(\vartheta^{(\ell, 1)}(t)\right)-\left(\dot{\vartheta}^{(\ell, 1)}(t)-\dot{\vartheta}^{(j, 1)}(t)\right) \cos \left(\vartheta^{(\ell, 1)}(t)-\vartheta^{(j, 1)}(t)\right)\right) \\
+\alpha_{0} \mu_{1} \sum_{\substack{\ell=2 \\
\ell \neq j}}^{N}\left(\sin \left(\vartheta^{(\ell, 1)}(t)\right)-\sin \left(\vartheta^{(\ell, 1)}(t)-\vartheta^{(j, 1)}(t)\right)\right)=0 .
\end{aligned}
$$

for $j=2, \ldots, N$. 
For the second-order filter, the model of the network is given by

$$
\begin{gathered}
\dddot{\vartheta}^{(j, 1)}(t)+\beta_{1} \ddot{\vartheta}^{(j, 1)}(t)+\beta_{0} \dot{\vartheta}^{(j, 1)}(t)+2 \mu_{1} \dot{\vartheta}^{(j, 1)}(t) \cos \left(\vartheta^{(j, 1)}(t)\right)+2 \alpha_{0} \mu_{1} \sin \left(\vartheta^{(j, 1)}(t)\right) \\
+\mu_{1} \sum_{\substack{\ell=2 \\
\ell \neq j}}^{N}\left(\dot{\vartheta}^{(\ell, 1)}(t) \cos \left(\vartheta^{(\ell, 1)}(t)\right)-\left(\dot{\vartheta}^{(\ell, 1)}(t)-\dot{\vartheta}^{(j, 1)}(t)\right) \cos \left(\vartheta^{(\ell, 1)}(t)-\vartheta^{(j, 1)}(t)\right)\right) \\
+\alpha_{0} \mu_{1} \sum_{\substack{\ell=2 \\
\ell \neq j}}^{N}\left(\sin \left(\vartheta^{(\ell, 1)}(t)\right)-\sin \left(\vartheta^{(\ell, 1)}(t)-\vartheta^{(j, 1)}(t)\right)\right)=0,
\end{gathered}
$$

for $j=2, \ldots, N$.

Therefore, (2.32) models the fully connected network considering first-order lead-lag node filters. Similarly, (2.33) represents the network with second-order node filters.In order to simplify the study of the equilibrium and its stability for (2.32) and (2.33),

the next section presents state space models for both cases.

\section{State Space Equations}

From (2.32), state equations can be established considering the phase and frequency errors, measured taking node 1 as reference, that is,

$$
\begin{aligned}
& x_{1}^{(m)}=\vartheta^{(j, 1)}(t), \\
& x_{2}^{(m)}=\dot{\vartheta}^{(j, 1)}(t),
\end{aligned}
$$

for $j=2, \ldots, N$ and $m=j-1$.

Then, considering a first-order lag-lead low-pass filter, state equations are

$$
\begin{aligned}
\dot{x}_{1}^{(m)}= & x_{2}^{(m)} \\
\dot{x}_{2}^{(m)}= & -\beta_{0} x_{2}^{(m)}-2 \mu_{1} x_{2}^{(m)} \cos \left(x_{1}^{(m)}\right)-2 \alpha_{0} \mu_{1} \sin \left(x_{1}^{(m)}\right) \\
& -\mu_{1} \sum_{\substack{n=1 \\
n \neq m}}^{N-1}\left(x_{2}^{(n)} \cos \left(x_{1}^{(n)}\right)-\left(x_{2}^{(n)}-x_{2}^{(m)}\right) \cos \left(x_{1}^{(n)}-x_{1}^{(m)}\right)\right) \\
& -\alpha_{0} \mu_{1} \sum_{\substack{n=1 \\
n \neq m}}^{N-1}\left(\sin \left(x_{1}^{(n)}\right)-\sin \left(x_{1}^{(n)}-x_{1}^{(m)}\right)\right),
\end{aligned}
$$

for $m=1,2, \ldots, N-1$. 
Defining a new state variable,

$$
x_{3}^{(m)}=\ddot{\vartheta}^{(j, 1)}(t),
$$

and by using (2.33), state equations considering a second-order low-pass filter are derived as

$$
\begin{aligned}
\dot{x}_{1}^{(m)}= & x_{2}^{(m)} \\
\dot{x}_{2}^{(m)}= & x_{3}^{(m)} \\
\dot{x}_{3}^{(m)}= & -\beta_{1} x_{3}^{(m)}-\beta_{0} x_{2}^{(m)}-2 \mu_{1} x_{2}^{(m)} \cos \left(x_{1}^{(m)}\right)-2 \alpha_{0} \mu_{1} \sin \left(x_{1}^{(m)}\right) \\
& -\mu_{1} \sum_{\substack{n=1 \\
n \neq m}}^{N-1}\left(x_{2}^{(n)} \cos \left(x_{1}^{(n)}\right)-\left(x_{2}^{(n)}-x_{2}^{(m)}\right) \cos \left(x_{1}^{(n)}-x_{1}^{(m)}\right)\right) \\
& -\alpha_{0} \mu_{1} \sum_{\substack{n=1 \\
n \neq m}}^{N-1}\left(\sin \left(x_{1}^{(n)}\right)-\sin \left(x_{1}^{(n)}-x_{1}^{(m)}\right)\right),
\end{aligned}
$$

for $m=1,2, \ldots, N-1$.

Expressions (3.2) and (3.4) describe the dynamics of the phase adjustments of a fullyconnected network depending on the node parameters $\mu_{1}, \alpha_{0}, \beta_{0}$ and $\beta_{1}$, considering firstorder lag-lead and second-order filters, respectively. These equations allow the research of the synchronous state stability conducted in the next section.

\section{Synchronous State Stability}

The fully connected PLL network reaches the synchronous state when all the frequency errors vanish. Furthermore, the robustness of this state under small perturbations gives an idea about the network steady state operation.

In this section, the local stability of the synchronous state, for the two different filter types, by using the Taylor series representing the vector fields given by (3.2) and (3.4) in a neighborhood of the synchronous state is studied.

Calculating the eigenvalues of the Jacobian of the vector fields that represents their linear part, conditions for local asymptotic stability of the synchronous state are derived [25].

\subsection{First-Order Lag-Lead Filter Analysis}

The equilibrium points of the state equations in (3.2) that correspond to the synchronous states of the network are

$$
\mathbf{x}^{*(m)}=\left[\begin{array}{ll}
k \pi & 0
\end{array}\right]^{T}
$$


where $k=0, \pm 1, \pm 2, \ldots$; and for $m=1,2, \ldots, N-1$, then

$$
\mathbf{x}^{*}=\left[\begin{array}{llll}
\mathbf{x}^{*(1) T} & \mathbf{x}^{*(2) T} & \cdots & \mathbf{x}^{*(N-1) T}
\end{array}\right]^{T} .
$$

For odd values of $k$, the equilibrium points are unstable for any parameter combination [17]. The stability of the equilibrium points corresponding to even values of $k$ can be studied by the eigenvalues of (4.3), considering the equilibrium point $\mathbf{x}^{*}$, with $k=0$ :

$$
\mathbf{A}=\left[\begin{array}{ccccc}
0 & 1 & \cdots & 0 & 0 \\
-\alpha_{0} N \mu_{1} & -\beta_{0}-N \mu_{1} & \cdots & 0 & 0 \\
\vdots & \vdots & \ddots & \vdots & \vdots \\
0 & 0 & \cdots & 0 & 1 \\
0 & 0 & \cdots & -\alpha_{0} N \mu_{1} & -\beta_{0}-N \mu_{1}
\end{array}\right]
$$

The characteristic polynomial of the matrix $\mathbf{A}$ is

$$
P(\lambda)=\left(\lambda^{2}+\left(\beta_{0}+N \mu_{1}\right) \lambda+\alpha_{0} N \mu_{1}\right)^{N-1},
$$

that presents $N-1$ repeated pair of roots given by

$$
\lambda=-\frac{\beta_{0}+N \mu_{1}}{2} \pm \frac{\sqrt{\left(\beta_{0}+N \mu_{1}\right)^{2}-4 \alpha_{0} N \mu_{1}}}{2}
$$

As all physically possible parameters in (4.5) are positive real numbers, these roots are either real negative or complex conjugated with negative real part. Consequently, for any number of nodes and any parameter combination, the fully connected PLL network with first-order lag-lead filters presents locally asymptotically stable synchronous states.

\subsection{Second-Order Filter Analysis}

The equilibrium points of the state equations in (3.4) are

$$
\mathbf{x}^{*(m)}=\left[\begin{array}{lll}
k \pi & 0 & 0
\end{array}\right]^{T},
$$

where $k=0, \pm 1, \pm 2, \ldots$; and for $m=1,2, \ldots, N-1$, then

$$
\mathbf{x}^{*}=\left[\begin{array}{llll}
\mathbf{x}^{*(1) T} & \mathbf{x}^{*(2) T} & \cdots & \mathbf{x}^{*(N-1) T}
\end{array}\right]^{T} .
$$


For odd values of $k$, the equilibrium points are unstable for any parameter combination [27]. The stability of the equilibrium points corresponding to even values of $k$ can be studied by the eigenvalues of (4.8), considering the equilibrium point $\mathbf{x}^{*}$, with $k=0$ :

$$
\mathbf{A}=\left[\begin{array}{ccccccc}
0 & 1 & 0 & \cdots & 0 & 0 & 0 \\
0 & 0 & 1 & \cdots & 0 & 0 & 0 \\
-\alpha_{0} N \mu_{1} & -\beta_{0}-N \mu_{1} & -\beta_{1} & \cdots & 0 & 0 & 0 \\
\vdots & \vdots & \vdots & \vdots & \ddots & \vdots & \vdots \\
0 & 0 & 0 & \cdots & 0 & 1 & 0 \\
0 & 0 & 0 & \cdots & 0 & 0 & 1 \\
0 & 0 & 0 & \cdots & -\alpha_{0} N \mu_{1} & -\beta_{0}-N \mu_{1} & -\beta_{1}
\end{array}\right] .
$$

The characteristic polynomial of the matrix $\mathbf{A}$ is

$$
\left.P(\lambda)=\left(\lambda^{3}+\beta_{1} \lambda^{2}+\beta_{0}+N \mu_{1}\right) \lambda+\alpha_{0} N \mu_{1}\right)^{N-1} .
$$

In order to verify if (4.9) presents roots with positive real part, Routh-Hurwitz criterion ([35]) is applied as follows:

$$
\begin{array}{c|cc}
\lambda^{3} & 1 & \beta_{0}+N \mu_{1} \\
\lambda^{2} & \beta_{1} & \alpha_{0} N \mu_{1} \\
& \frac{\beta_{1}\left(\beta_{0}+N \mu_{1}\right)-\alpha_{0} N \mu_{1}}{\beta_{1}} & \\
\lambda^{0} & \alpha_{0} N \mu_{1}
\end{array}
$$

As all physically possible parameters are positive real numbers, the roots of (4.9) are either real negative or complex conjugated with negative real part only if

$$
N<\frac{\beta_{1} \beta_{0}}{\mu_{1}\left(\alpha_{0}-\beta_{1}\right)}
$$

Consequently, (4.11) gives a superior limit for the number of nodes in a fully connected PLL network with second-order node filters. Above this value, all the synchronous states of the network are unstable

\section{Conclusions}

fully connected PLL networks can have their performance improved by choosing filters different from first-order lag low-pass. If first-order lag-lead low-pass are chosen, the synchronous states of the network are locally asymptotically stable for any number of nodes. The adjustment of the parameters $\alpha_{0}$ and $\beta_{0}$ only changes the transient responses, allowing the desired bandwidth. 
If a second-order low pass is used, in order to further improve the transient response, (4.11) establishes a superior limitation for the number of nodes of the network.

\section{References}

[1] S. Bregni, Synchronization of Digital Networks, John Wiley \& Sons, Chichester, UK, 1st edition, 2002.

[2] W. C. Lindsey, F. Ghazvinian, W. C. Hagmann, and K. Dessouky, "Network synchronization," Proceedings of the IEEE, vol. 3, no. 10, pp. 1445-1467, 1985.

[3] H. Meyr and G. Ascheid, Synchronization in Digital Communications Phase-Frequency-Locked Loops, and Amplitude Control, vol. 1, John Wiley \& Sons, Chichester, UK, 1990.

[4] J. C. Bellamy, "Digital network synchronization," IEEE Communications Magazine, vol. 33, no. 4, pp. 70-83, 1995.

[5] M. Saint-Laurent and M. Swaminathan, "A multi-PLL clock distribution architecture for gigascale integration," in Proceedings of IEEE Computer Society Workshop on VLSI (IWV'01), pp. 30-35, Orlando, Fla, USA, May 2001.

[6] G. A. Pratt and J. Nguyen, "Distributed synchronous clocking," IEEE Transactions on Parallel and Distributed Systems, vol. 6, no. 3, pp. 314-328, 1995.

[7] V. Gutnik and A. P. Chandrakasan, "Active GHz clock network using distributed PLLs," IEEE Journal of Solid-State Circuits, vol. 35, no. 11, pp. 1553-1560, 2000.

[8] J. R. C. Piqueira, F. M. Orsatti, and L. H. A. Monteiro, “Computing with phase locked loops: choosing gains and delays," IEEE Transactions on Neural Networks, vol. 14, no. 1, pp. 243-247, 2003.

[9] L. H. A. Monteiro, N. C. F. Canto, J. G. Chaui-Berlinck, F. M. Orsatti, and J. R. C. Piqueira, "Global and partial synchronism in phase-locked loop networks," IEEE Transactions on Neural Networks, vol. 14, no. 6, pp. 1572-1575, 2003.

[10] F. C. Hoppensteadt and E. M. Izhikevich, "Pattern recognition via synchronization in phase-locked loop neural networks," IEEE Transactions on Neural Networks, vol. 11, no. 3, pp. 734-738, 2000.

[11] H. Inose, H. Fujisaki, and T. Saito, “Theory of mutually synchronized systems," Electronics Letters, vol. 2, no. 3, pp. 96-97, 1966.

[12] A. Gersho and B. J. Karafin, "Mutual synchronization of geographically separated oscillators," The Bell System Technical Journal, vol. 45, pp. 1689-1704, 1996.

[13] M. Karnaugh, "A model for the organic synchronization of communication systems," The Bell System Technical Journal, vol. 45, pp. 1705-1735, 1966.

[14] M. W. Williard, "Analysis of a system of mutually synchronized oscillators," IEEE Transactions on Communication Technology, vol. 18, no. 5, pp. 467-483, 1970.

[15] W. C. Lindsey and A. V. Kantak, "Network synchronization of random signals," IEEE Transactions on Communications Systems, vol. 28, no. 8, part 2, pp. 1260-1266, 1980.

[16] J. R. C. Piqueira, M. Q. Oliveira, and L. H. A. Monteiro, "Synchronous state in a fully connected phaselocked loop network," Mathematical Problems in Engineering, vol. 2006, Article ID 52356, 12 pages, 2006.

[17] J. R. C. Piqueira, M. Q. de Oliveira, and L. H. A. Monteiro, "Linear approach for synchronous state stability in fully connected PLL networks," Mathematical Problems in Engineering, vol. 2008, Article ID 364084, 13 pages, 2008.

[18] J. R. C. Piqueira and A. Z. Caligares, “Double-frequency jitter in chain master-slave clock distribution networks: comparing topologies," Journal of Communications and Networks, vol. 8, no. 1, pp. 8-12, 2006.

[19] J. R. C. Piqueira, S. A. Castillo-Vargas, and L. H. A. Monteiro, "Two-way master-slave double-chain networks: limitations imposed by linear master drift for second order PLLs as slave nodes," IEEE Communications Letters, vol. 9, no. 9, pp. 829-831, 2005.

[20] J. R. C. Piqueira, A. Z. Caligares, and L. H. A. Monteiro, “Double-frequency jitter figures in masterslave PLL networks," International Journal of Electronics and Communications, vol. 61, no. 10, pp. 678683, 2007.

[21] R. E. Best, Phase-Locked Loops. Design, Simulation and Applications, McGraw-Hill, New York, NY, USA, 2003.

[22] J. R. C. Piqueira and L. H. A. Monteiro, "All-pole phase-locked loops: calculating lock-in range by using Evan's root-locus," International Journal of Control, vol. 79, no. 7, pp. 822-829, 2006.

[23] J. R. C. Piqueira and L. H. A. Monteiro, "Considering second-harmonic terms in the operation of the phase detector for second-order phase-locked loop," IEEE Transactions on Circuits and Systems I, vol. 50, no. 6, pp. 805-809, 2003. 
[24] J. R. C. Piqueira, E. Y. Takada, and L. H. A. Monteiro, "Analyzing the effect of the phase-jitter in the operation of second order phase-locked loops," IEEE Transactions on Circuits and Systems II, vol. 52, no. 6, pp. 331-335, 2005.

[25] J. Guckenheimer and P. Holmes, Nonlinear Oscillations, Dynamical Systems, and Bifurcations of Vector Fields, vol. 42 of Applied Mathematical Sciences, Springer, New York, NY, USA, 1983.

[26] L. H. A. Monteiro, D. N. F. Filho, and J. R. C. Piqueira, "Bifurcation analysis for third-order phaselocked loops," IEEE Signal Processing Letters, vol. 11, no. 5, pp. 494-496, 2004.

[27] J. R. C. Piqueira, "Using bifurcations in the determination of lock-in ranges for third-order phaselocked loops," Communications in Nonlinear Science and Numerical Simulation, vol. 14, no. 5, pp. 2328 2335, 2009.

[28] J. R. C. Piqueira, C. N. Marmo, and L. H. A. Monteiro, “Using central manifold theorem in the analysis of master-slave synchronization networks," Journal of Communications and Networks, vol. 6, no. 3, pp. 197-202, 2004.

[29] J. R. C. Piqueira and M. D. C. Freschi, "Models for master-slave clock distribution networks with third-order phase-locked loops," Mathematical Problems in Engineering, vol. 2007, Article ID 18609, 17 pages, 2007.

[30] F. M. Orsatti, R. Carareto, and J. R. C. Piqueira, "Mutually connected phase-locked loop networks: dynamical models and design parameters," IET Circuits, Devices and Systems, vol. 2, no. 6, pp. 495508, 2008.

[31] R. Carareto, F. M. Orsatti, and J. R. C. Piqueira, "Reachability of the synchronous state in a mutually connected PLL network," International Journal of Electronics and Communications. In press.

[32] A. A. Ferreira, A. M. Bueno, and J. R. C. Piqueira, "Modeling and measuring double-frequency jitter in one-way master-slave networks," Communications in Nonlinear Science and Numerical Simulation, vol. 14, no. 5, pp. 1854-1860, 2009.

[33] T. Kailath, Linear Systems, Prentice-Hall, Englewood Cliffs, NJ, USA, 1980, Prentice-Hall Information and System Sciences Serie.

[34] F. M. Gardner, Phaselock Techniques, John Wiley \& Sons, Chichester, UK, 3rd edition, 2005.

[35] K. Ogata, Modern Control Engineering, Prentice-Hall, Englewood Cliffs, NJ, USA, 2002. 


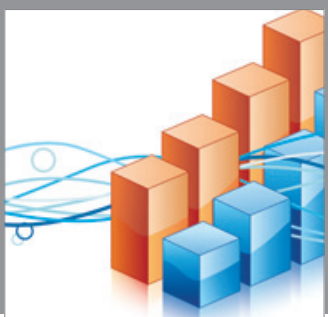

Advances in

Operations Research

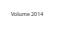

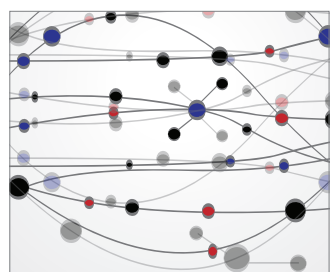

\section{The Scientific} World Journal
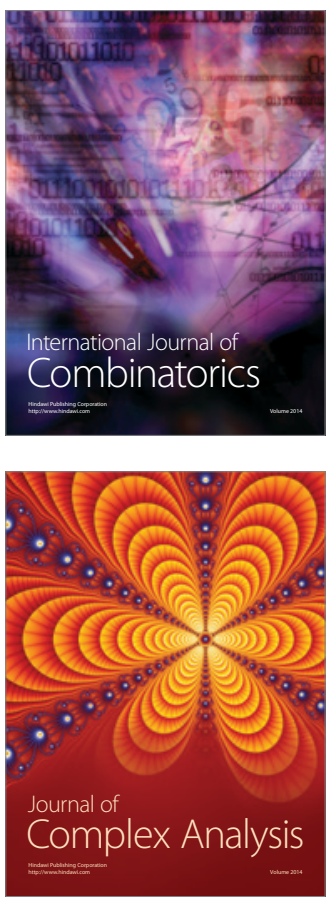

International Journal of

Mathematics and

Mathematical

Sciences
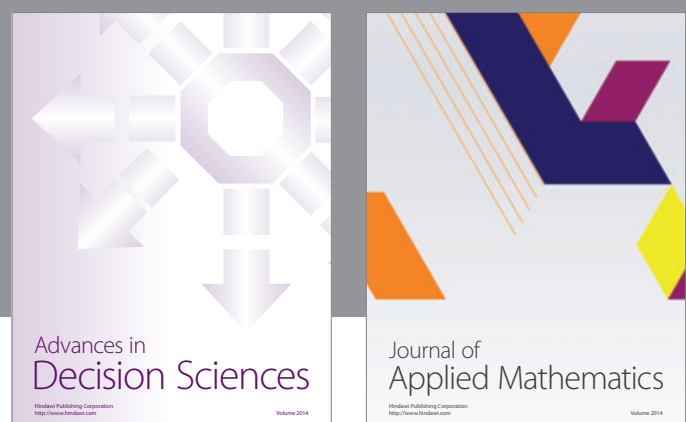

Journal of

Applied Mathematics
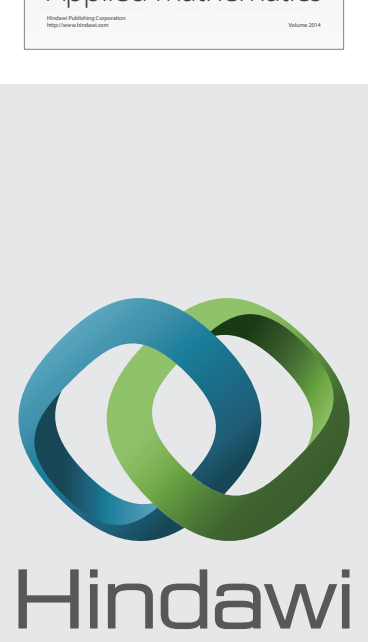

Submit your manuscripts at http://www.hindawi.com
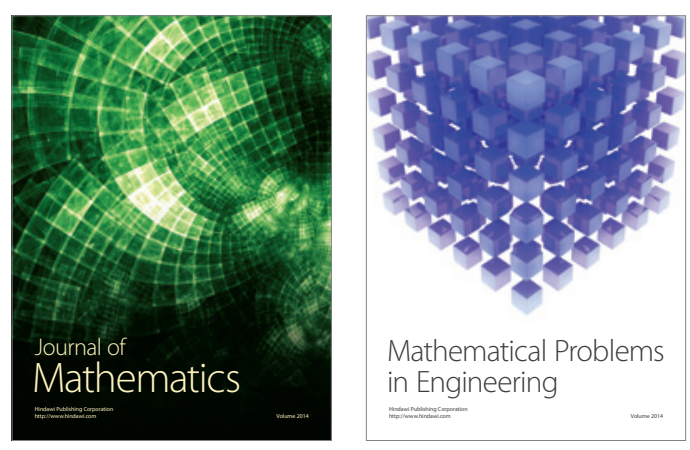

Mathematical Problems in Engineering
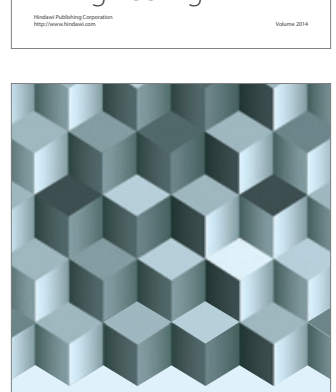

Journal of

Function Spaces
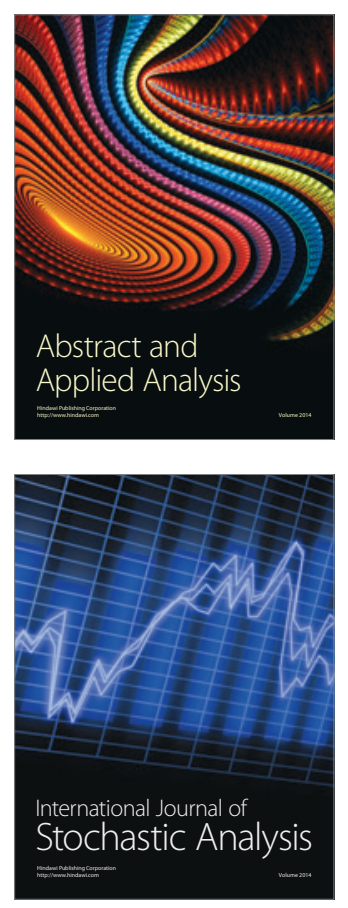

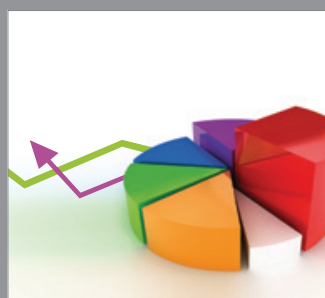

ournal of

Probability and Statistics

Promensencen
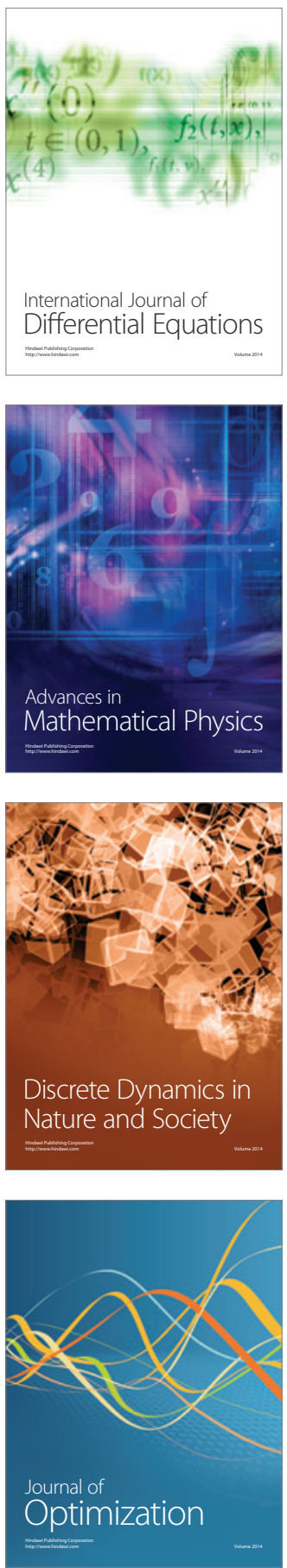